\title{
Fat chance for longevity
}

\author{
Marina Kniazeva ${ }^{1}$ and Min Han \\ Howard Hughes Medical Institute, Department of Molecular, Cell, and Developmental Biology, University of Colorado \\ at Boulder, Boulder, Colorado 80309, USA
}

The health benefits of specific fatty acids and physiological roles of fat metabolism are important subjects that are still poorly understood. In this issue of Genes \& Development, O'Rourke and colleagues (pp. 429-440) uncovered a role for lipase-generated $\omega-6$ fatty acids in promoting autophagy and, consequently, life span extension under both fed and fasting conditions. The impact of this finding is discussed with regard to the nutritional value of $\omega-6$ fatty acids and regulatory functions of fat metabolism beyond its well-known role in energy storage.

The study by O'Rourke et al. (2013) in this issue of Genes \& Development is a significant step toward understanding the complex and obscure effect of fat metabolism on animal longevity. In a previous study (Wang et al. 2008), this group reported that lipid hydrolysis involving a specific lipase is a critical cellular event connecting germline stem cell activity and life span in Caenorhabditis elegans. Now, O'Rourke et al. (2013) describe a number of molecular and cellular events underlying the functional relationship between starvation, fat hydrolysis, and longevity. They suggest a mechanism by which enrichment in specific fatty acids from fat hydrolysis promotes life span extension by activating autophagy and provide evidence that an analogous model may be applicable to mammals (Fig. 1A).

Specifically, O'Rourke et al. (2013) show that an increase in the expression of the lipl-4 gene, encoding one of the predicted fat hydrolyzing lipases in C. elegans (Pistillo et al. 1998; Wang et al. 2008; Lapierre et al. 2011, 2012), results in increased levels of the 20-carbon $\omega-3$ and $\omega-6$ polyunsaturated fatty acids (PUFAs) eicosapentaenoic acid (EPA) and arachidonic acid (AA), respectively. The elevated levels of AA, but not EPA, are sufficient to activate autophagy. Known physiological impacts of autophagy include supplying essential nutrients, saving energy at times when food consumption is terminated, and promoting life span extension (Levine and Klionsky 2004; Cuervo 2008; Rabinowitz and White 2010; Rubinsztein

[Keywords: C. elegans; fasting; lipid; autophagy; polyunsaturated fatty acids (PUFA); longevity]

${ }^{1}$ Corresponding author

E-mail marina.kniazeva@colorado.edu

Article is online at http://www.genesdev.org/cgi/doi/10.1101/gad.214189.113. et al. 2011). By showing that AA can activate autophagy and extend longevity without caloric restriction, O'Rourke et al. (2013) argue that the increased levels of LIPL-4 and AA are the causal events in life span extension.

In humans, AA and EPA are derivatives of essential linoleic $(\omega-6)$ and linolenic $(\omega-3)$ acids, major components of plant and algae lipids, respectively. AA and EPA are 20-carbon chain FAs with different numbers and positions of double bonds along the carbon backbone. The principal difference, though, is the position of the first (counting from the terminal methyl group) double bond at the third $(\omega-3)$ and sixth $(\omega-6)$ positions. It appears that it is the positioning of the first double bond that determines the impact of PUFAs on biological processes. Both $\omega-3$ and $\omega-6$ PUFAs have a huge range of essential biological functions as free FAs or as components of more complex lipids, such as triacylglycerol (TAG) and phospholipids (PLs). They also are precursors of biologically active eicosanoids (prostaglandins, leukotrients, etc.) and endocanabinoids (2-arachidonoylglycerol and $\mathrm{N}$-arachidonoylethanolamine).

$\omega-3$ and $\omega-6$ PUFAs are essential FAs that must be obtained from the diet. It was estimated that because of an increase in plant oil usage and a reduction in fish consumption over the past thousands of years, the balance between $\omega-3$ and $\omega-6$ in the human diet, and subsequently in human lipids, has considerably changed (Simopoulos et al. 2000; Kris-Etherton et al. 2002; Simopoulos 2002). Results of epidemiological studies revealed a correlation between an increased $\omega-6 / \omega-3$ ratio and instances of obesity, metabolic syndrome, cardiovascular diseases, stroke, cancer, mental disorders, and more (Simopoulos 2002). One of the explanations of such an effect could be a strong proinflammatory effect of AA. Indeed, a body of work has suggested that reducing AA and increasing $\omega-3$ PUFA could reduce risks of pathological conditions.

Naturally, there has been a great deal of interest in using dietary supplements, such as fish oil or $\omega-3$ FA pills, or choosing an $\omega$-3-rich "Mediterranean" diet over the typical "Western" one to alter the ratio for health benefits. However, the effects of PUFA supplementations have been challenged (Harris et al. 2009; Rizos et al. 2012), and future studies in the field will likely sway the conventional view on the subject. For example, a recent study based on systematic evaluation and meta-analysis suggested that $\omega-3$ PUFA supplementation was not associated with a lower risk of all-cause mortality, including 


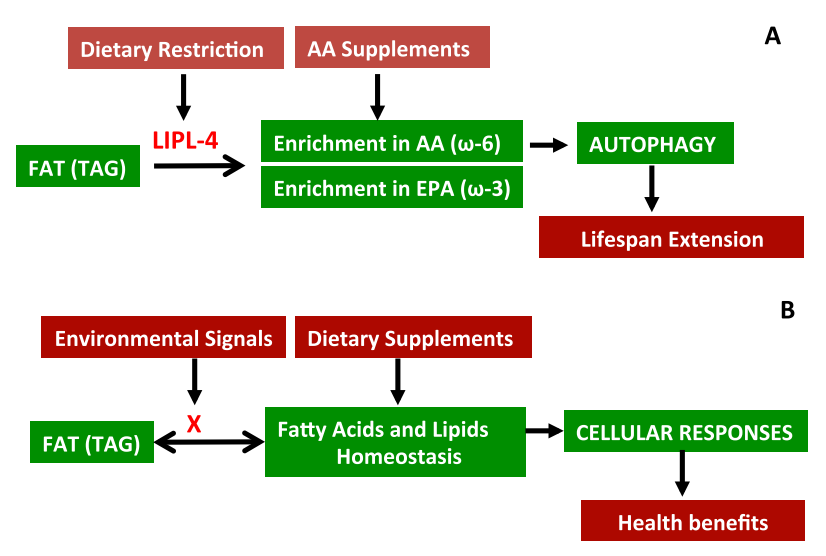

Figure 1. Fat metabolites mediate important cellular functions in response to environmental changes. $(A)$ Roles of lipase-mediated fat metabolism and AA supplements on life span extension under fed and fasting conditions, based on O'Rourke et al. (2013). Dietary restriction promotes expression of LIPL-4 and possibly other lipases that hydrolyze fat or other lipids, which in turn results in the enrichment of certain $\omega-3$ and $\omega-6$ PUFAs (EPA and AA, respectively). High level of AA, but not EPA, induces autophagy and promotes longevity. Although LIPL-4 was predicted to be a TAG lipase, it is possible that its major substrates include other lipids, such as phospholipids that could be involved in the fasting response pathway. $(B)$ A generalized model illustrating the impact of fat/TAG metabolism on cellular processes and animal health. (X) Factors, such as lipases, that play critical roles in regulating fat metabolism and FA homeostasis in response to environmental changes or other signals.

cardiac death, sudden death, myocardial infarction, or stroke (Rizos et al. 2012). Furthermore, several studies have already suggested the beneficial effect of $\omega-6$ PUFA during fasting conditions (see the Discussion in O'Rourke et al. 2013). By showing that AA, but not EPA, stimulates autophagy and promotes life span extension, O'Rourke et al. (2013) indicated a mechanism by which $\omega-6$ PUFAs may positively impact human health.

In humans, stimulation of autophagy has been shown to have anti-aging effects, while reduced autophagy has been linked to premature aging (Madeo et al. 2010). A failure or misuse of autophagy has been associated with numerous pathological conditions, including neurodegenerative disorders, inflammation, and cancer (Kuballa and Xavier 2010). Activation of autophagy and its positive contribution to extension of life span in model organisms have been extensively reviewed (Rubinsztein et al. 2011). The study by O'Rourke et al. (2013) suggests that supplementation of $\omega-6 / \omega-3$ PUFAs (or their derivatives) could be used to manipulate autophagy to prevent or treat disorders and enhance overall wellness. It would be interesting to see whether dietary $\omega-6$ PUFA could make it to the list of proautophagy and prolongevity dietary supplements.

This study also promotes a seemingly simple but often overlooked message regarding fat as a metabolite: It has vital physiological value well beyond its contribution to energy storage. Enzymatic conversion of fat (in this case, with the starvation-inducible LIPL-4 and/or other lipases) may shift the FA composition toward increased levels of
PUFA and stimulate protective mechanisms such as autophagy (Fig. 1). How does the hydrolysis of fat result in increased PUFAs? What are the breakdown products of the LIPL-4 and possibly other lipases involved in the process? These questions remind us to take a closer look at fat as a unique group of lipid molecules.

Fat is easily observable, directly and indirectly, and is likely the most talked about of all the lipids. At certain times and in specific cultures, the accumulation of human body fat has been considered as a sign of health and wealth, while at other times it has been perceived in the opposite way. An interest in understanding the chemical structure of fat is as ancient as biology. The presence of an acidic component in fat was reported by Tachenius Otto in 1673, the same year that Antonie Van Leeuwenhoek began to publish his first observations of microorganisms made under his microscope (Partington 1961; Krebs 2008). More than 100 years passed by before glycerol was identified by Carl Wilhelm Scheele in 1779 and shown to be a principal component of fat by Michel Eugène Chevreul in 1813 (Partington 1961). At roughly the same time, scientists recognized a cell as the unit of all living tissues (Krebs 2008).

Chemically, fat is defined as TAG: esters of glycerol and three fatty acids (FAs). The known biochemical function of fat is to store and supply energy to cells and tissues when it is needed. This function is carried out by lipases, which hydrolyze ester bonds and produce FAs and glycerol that are available for oxidation and glycolysis, respectively. This simplified and utilitarian view of fat may contribute to its perception as a relatively dull molecule, especially when compared with more glamorous regulatory lipids such as steroids, prostaglandins, and even FAs themselves. Recent research, however, has suggested that fat may have more physiological uses than as a "simple" storage molecule.

It is hard to ignore the elaborate complexity associated with fat metabolism. In nature, TAGs are present as a mixture of TAG species that differ according to their FA content. In TAGs, FAs vary from medium chain saturated FA (SFA) to long chain PUFA occupying a specific stereo position (stereospecific numbering, sn) on the glycerol backbone. For example, TAGs of adipose tissue in animals frequently have an SFA at the $s n-1$ position and an unsaturated FA at the sn-2 position (Brockerhoff et al. 1966). The factors contributing to a particular design of TAG species remain obscure, but the effect of FA stereopositioning has been thoroughly studied, especially with regard to its role in the absorption of dietary fats.

In the intestine, dietary fat or TAG is broken down into monoacylglycerol (MAG) before its absorption by enterocytes. This hydrolysis is carried out sequentially by gastric and pancreatic lipases sensitive to FA composition in such a way that FA at the sn-2 position is preferably preserved. MAG and free FAs then use different cellular entryways (passive or assisted) and eventually re-esterify with each other or incur alternative metabolic fates within the cells. Thus, stereopositioning of FAs in TAGs may determine the metabolism of their catabolites. Indeed, it was shown that absorption of $\omega-3$ and $\omega-6$ PUFAs 
from fish TAGs is higher when they occupy sn-2 position (Amate et al. 1999; Ramirez et al. 2001). TAG reassembly inside the intestinal cells is less clear but eventually profoundly affects TAG, FA, PL, and cholesterol metabolism (for review, see Karupaiah and Sundram 2007).

Utilization of TAGs as a fuel also appears to be complicated and even counterintuitive. It was shown that $20 \%-50 \%$ of FAs are re-esterified in fasting animals. The re-esterification takes place, although at low rates, even during long periods of fasting (Weber and Reidy 2012). It happens in either the same cells where they were released by lipolysis or distal cells where they were transported (Weber and Reidy 2012). Inconsistent with a role of TAG as an energy source, TAG re-esterification continues to consume ATP (the ultimate energy molecule) at times when efficient energy expenditure is essential for survival. It is conceivable that a remodeling of TAGs and possibly other lipids has essential biological functions that are yet to be understood.

Taking into account the molecular diversity of TAGs and the complex chemical conversions involved in metabolism of these lipid species, the absence of a correlation between overall fat levels and life span in animals (for review, see Ackerman and Gems 2012; Hou and Taubert 2012 ) is not so surprising. It is plausible that variations in the TAG structure and selectivity of specific lipases (and other enzymes acting on TAG), being both substrate- and tissue-specific, may contribute to the generation of metabolites with distinct impacts on cellular processes and physiological functions (Fig. 1B). The expression levels or the activities of these TAG metabolic enzymes may be subjected to regulation by environmental cues and/or internal signals. The O'Rourke et al. (2013) study, using both C. elegans and mammalian tissue culture as model systems, presents an excellent case for a regulatory role of TAG metabolism. The specific breakdown products generated by LIPL-4 and possibly other lipases stimulate a whole array of events: enrichment of $\omega-6$ PUFA, transcriptional up-regulation of the FA-binding proteins, activation of autophagy, and finally, extension of life span during fasting, supporting the hypothesis that these lipid metabolites act as signals of food scarcity.

Interestingly, an important component of autophagy is lipophagy, a selective form of catabolic lipid conversions (Singh et al. 2009). However, the mechanism by which TAGs and other lipids are recognized as substrates and targeted for degradation remains unclear. It has been proposed that in addition to regulating lipid content and meeting energy demands, the ability of lipophagy to selectively break down lipids serves as yet unknown functions (for review, see Liu and Czaja 2013). Whether or how lipophagy is involved in the extension of life span would be an interesting question to follow.

Dissecting the contribution of lipid structures to the selectivity of biochemical processes and their impact on human health will require a more precise, high-resolution level of analysis. Although advances have already been made in this direction using model organisms (e.g., Brock et al. 2007; O'Rourke et al. 2009; Shmookler Reis et al. 2011; Vrablik and Watts 2012; Liu and Czaja 2013), future genetic studies empowered by analytical biochemistry methods promise a great breakthrough in this important field of research.

\section{Acknowledgments}

We thank Aileen Sewell for comments on the manuscript.

\section{References}

Ackerman D, Gems D. 2012. The mystery of C. elegans aging: An emerging role for fat. Distant parallels between C. elegans aging and metabolic syndrome? BioEssays 34: 466-471.

Amate L, Ramirez M, Gil A. 1999. Positional analysis of triglycerides and phospholipids rich in long-chain polyunsaturated fatty acids. Lipids 34: 865-871.

Brock TJ, Browse J, Watts JL. 2007. Fatty acid desaturation and the regulation of adiposity in Caenorhabditis elegans. Genetics 176: 865-875.

Brockerhoff H, Hoyle RJ, Wolmark N. 1966. Positional distribution of fatty acids in triglycerides of animal depot fats. Biochim Biophys Acta 116: 67-72.

Cuervo AM. 2008. Autophagy and aging: Keeping that old broom working. Trends Genet 24: 604-612.

Harris WS, Mozaffarian D, Rimm E, Kris-Etherton P, Rudel LL, Appel LJ, Engler MM, Engler MB, Sacks F. 2009. $\omega-6$ Fatty acids and risk for cardiovascular disease: A science advisory from the American Heart Association Nutrition Subcommittee of the Council on Nutrition, Physical Activity, and Metabolism; Council on Cardiovascular Nursing; and Council on Epidemiology and Prevention. Circulation 119: 902-907.

Hou NS, Taubert S. 2012. Function and regulation of lipid biology in Caenorhabditis elegans aging. Front Physiol 3: 143.

Karupaiah T, Sundram K. 2007. Effects of stereospecific positioning of fatty acids in triacylglycerol structures in native and randomized fats: A review of their nutritional implications. Nutr Metab 4: 16.

Krebs RE. 2008. Encyclopedia of scientific principles, laws, and theories. Greenwood Press, Westport, Conn.

Kris-Etherton PM, Harris WS, Appel LJ. 2002. Fish consumption, fish oil, $\omega-3$ fatty acids, and cardiovascular disease. Circulation 106: 2747-2757.

Kuballa P, Xavier RJ. 2010. Failure and exploitation of autophagy in human pathologies-cellular integrity between inflammation, infection, and cell survival. Semin Immunopathol 32: 319-322.

Lapierre LR, Gelino S, Melendez A, Hansen M. 2011. Autophagy and lipid metabolism coordinately modulate life span in germline-less C. elegans. Curr Biol 21: 1507-1514.

Lapierre LR, Melendez A, Hansen M. 2012. Autophagy links lipid metabolism to longevity in C. elegans. Autophagy 8: 144-146.

Levine B, Klionsky DJ. 2004. Development by self-digestion: Molecular mechanisms and biological functions of autophagy. Dev Cell 6: 463-477.

Liu K, Czaja MJ. 2013. Regulation of lipid stores and metabolism by lipophagy. Cell Death Differ 20: 3-11.

Madeo F, Tavernarakis N, Kroemer G. 2010. Can autophagy promote longevity? Nat Cell Biol 12: 842-846.

O'Rourke EJ, Soukas AA, Carr CE, Ruvkun G. 2009. C. elegans major fats are stored in vesicles distinct from lysosomerelated organelles. Cell Metab 10: 430-435.

O'Rourke EJ, Kuballa P, Xavier R, Ruvkun G. 2013. w-6 Polyunsaturated fatty acids extend life span through the activa- 
tion of autophagy. Genes Dev (this issue). doi: 10.1101/ gad.205294.112.

Partington JR. 1961. A history of chemistry. Macmillan, London.

Pistillo D, Manzi A, Tino A, Boyl PP, Graziani F, Malva C. 1998. The Drosophila melanogaster lipase homologs: A gene family with tissue and developmental specific expression. J Mol Biol 276: 877-885.

Rabinowitz JD, White E. 2010. Autophagy and metabolism. Science 330: 1344-1348.

Ramirez M, Amate L, Gil A. 2001. Absorption and distribution of dietary fatty acids from different sources. Early Hum Dev 65: S95-S101.

Rizos EC, Ntzani EE, Bika E, Kostapanos MS, Elisaf MS. 2012. Association between $\omega-3$ fatty acid supplementation and risk of major cardiovascular disease events: A systematic review and meta-analysis. JAMA 308: 1024-1033.

Rubinsztein DC, Marino G, Kroemer G. 2011. Autophagy and aging. Cell 146: 682-695.

Shmookler Reis RJ, Xu L, Lee H, Chae M, Thaden JJ, Bharill $\mathrm{P}_{,}$ Tazearslan C, Siegel E, Alla R, Zimniak P, Ayyadevara S. 2011. Modulation of lipid biosynthesis contributes to stress resistance and longevity of C. elegans mutants. Aging 3: $125-144$.

Simopoulos AP. 2002. The importance of the ratio of $\omega-6 / \omega-3$ essential fatty acids. Biomed Pharmacother 56: 365-379.

Simopoulos AP, Leaf A, Salem N Jr. 2000. Workshop statement on the essentiality of and recommended dietary intakes for $\omega-6$ and $\omega-3$ fatty acids. Prostaglandins Leukot Essent Fatty Acids 63: 119-121.

Singh R, Kaushik S, Wang Y, Xiang Y, Novak I, Komatsu M, Tanaka K, Cuervo AM, Czaja MJ. 2009. Autophagy regulates lipid metabolism. Nature 458: 1131-1135.

Vrablik TL, Watts JL. 2012. Emerging roles for specific fatty acids in developmental processes. Genes Dev 26: 631-637.

Wang MC, O'Rourke EJ, Ruvkun G. 2008. Fat metabolism links germline stem cells and longevity in C. elegans. Science 322: 957-960.

Weber JM, Reidy SP. 2012. Extending food deprivation reverses the short-term lipolytic response to fasting: Role of the triacylglycerol/fatty acid cycle. J Exp Biol 215: 1484-1490. 


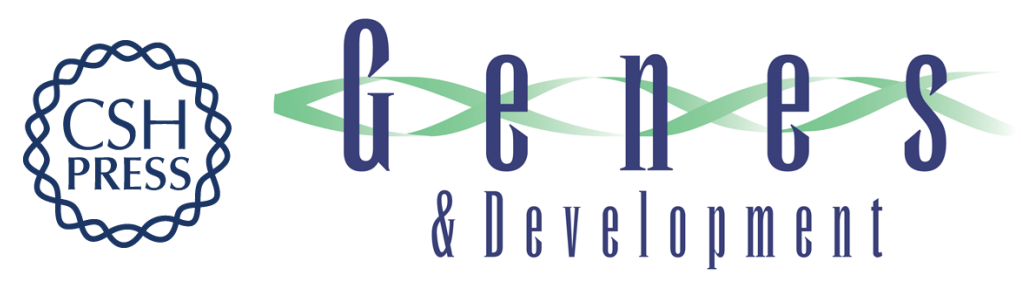

\section{Fat chance for longevity}

Marina Kniazeva and Min Han

Genes Dev. 2013, 27:

Access the most recent version at doi:10.1101/gad.214189.113

\section{Related Content É-6 Polyunsaturated fatty acids extend life span through the activation of autophagy \\ Eyleen J. O'Rourke, Petric Kuballa, Ramnik Xavier, et al. \\ Genes Dev. February, 2013 27: 429-440 \\ References This article cites 28 articles, 7 of which can be accessed free at: \\ http://genesdev.cshlp.org/content/27/4/351.full.html\#ref-list-1 \\ Articles cited in: \\ http://genesdev.cshlp.org/content/27/4/351.full.htmI\#related-urls \\ License \\ Email Alerting Receive free email alerts when new articles cite this article - sign up in the box at the top \\ Service right corner of the article or click here.}

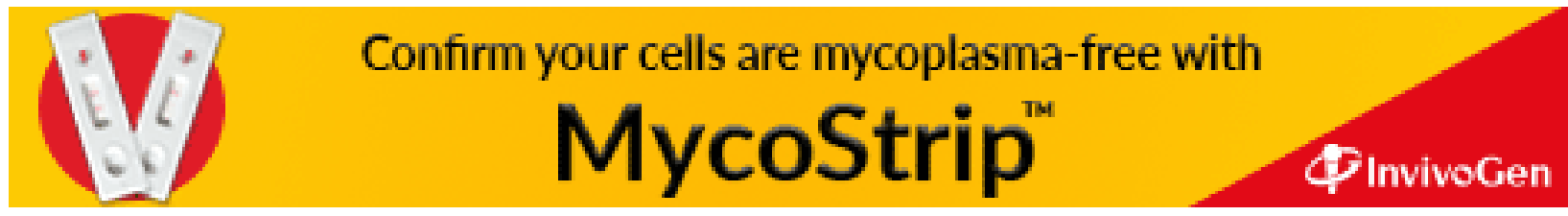

